(6)

OPEN ACCESS

\title{
CASPR2 autoantibodies are raised during pregnancy in mothers of children with mental retardation and disorders of psychological development but not autism
}

\author{
Ester Coutinho, ${ }^{1}$ Leslie Jacobson, ${ }^{1}$ Marianne Giørtz Pedersen, ${ }^{2,3}$ \\ Michael Eriksen Benros, ${ }^{2,3,4}$ Bent Nørgaard-Pedersen, ${ }^{5}$ Preben Bo Mortensen, 2,3,6 \\ Paul J Harrison, ${ }^{7,8}$ Angela Vincent ${ }^{1}$
}

For numbered affiliations see end of article.

\section{Correspondence to} Professor Angela Vincent, Nuffield Department of Clinical Neurosciences, John Radcliffe Hospital, Oxford OX3 9DU, UK angela.vincent@ndcn.ox.ac.uk

Received 9 November 2016 Revised 20 March 2017 Accepted 24 March 2017 Published Online First 1 June 2017

\section{Linked}

- http://dx.doi.org/10.1136/ jnnp-2017-315724

\section{CrossMark}

\section{To cite: Coutinho $\mathrm{E}$} Jacobson L, Pedersen MG, et al. J Neurol Neurosurg Psychiatry 2017;88:718-721.

\section{ABSTRACT}

Background, Methods and Objectives Maternal autoantibodies to neuronal proteins may be one cause of neurodevelopmental disorders. This exploratory study used the Danish archived midgestational sera and their nationwide registers to search for antibodies to the $\mathrm{N}$-methyl-D-aspartate receptor (NMDAR) and contactinassociated protein-like 2 (CASPR2) in maternal sera, and to relate them to subsequent psychiatric diagnoses in the woman or her child.

Results In a sample of 192 women, there was no association between antibody status and subsequent psychosis in the mothers. However, NMDAR antibodies $(n=4)$ or CASPR2 antibodies $(n=1)$ were identified in $5 / 11(45.5 \%)$ women whose children were given a diagnosis of mild or unspecified mental retardation or disorders of psychological and motor development (collectively abbreviated as mental retardation and/ or disorders of psychological development (MR/DPD)) compared with $9 / 176(5.1 \%)$ of the remaining mother $(p<0.001)$. These findings were followed up in a specifically selected cohort, in which CASPR2 antibodies were detected in 7/171 (4.1\%) mothers of MR/DPD progeny, compared with only $1 / 171(0.6 \%)$ control mother $(p=0.067)$. The combined sample showed a significantly higher frequency of CASPR2 antibodies in mothers of MD/DPD children $(p=0.01)$. These autoantibodies were not increased in mothers of children with autistic spectrum disorder.

Conclusions These findings complement the known roles of CASPR2 in brain development, and warrant further epidemiological and experimental studies to clarify the role of CASPR2 and possibly other antibodies in neurodevelopmental disorders.

\section{INTRODUCTION}

A role for autoantibodies is increasingly apparent for several neuropsychiatric phenotypes, including encephalitis, epilepsy and psychosis. ${ }^{1}$ Prominent among the identified neuronal surface targets are the N-methyl-D-aspartate receptor (NMDAR) and contactin-associated protein-like 2 (CASPR2). Meanwhile, transfer of immunoglobulin $\mathrm{G}$ ( $\mathrm{IgG}$ ) antibodies from mother to the fetus during pregnancy may be one cause of neurodevelopmental changes in their offspring. ${ }^{23}$ However, the identity of specific extracellular targets, the importance of autoantibodies as risk factors and the range of disorders in affected children are undetermined.

NMDAR antibodies have been reported in women with postpartum psychosis, ${ }^{4}$ and such women may be at higher risk for children with neurodevelopmental disorders, ${ }^{5}$ so we first asked whether neuronal surface antibodies were raised in mothers with subsequent psychosis and whether they were associated with an increased incidence of neurodevelopmental disorders in their children. Based on the results, we then looked at specifically selected samples for CASPR2 and NMDAR antibodies in mothers of children with mild or unspecified mental retardation and/or disorders of psychological development (MR/DPD). For comparison, we also tested 190 samples from mothers of children given a diagnosis of autism and control mothers.

\section{METHODS}

\section{Cohorts}

The pregnancy screening biobank at the State Serum Institute stores serum samples taken from more than 100000 women who gave birth in five Danish counties from 1977 to 1997. In Denmark, all residents can be linked to clinical registries and biobanks. We used the nationwide Danish Psychiatric Central Research Register (DPCRR) to identify the following samples in the biobank. ${ }^{6-8}$

Cohort A: This included first trimester mothers with a first diagnosis of schizophrenia spectrum disorder (International Classification of Diseases (ICD)-8: 295, 297, 298.39, 301.83; ICD-10: F20F29) at some time after delivery $(n=94$, cases $)$ and mothers without a history of schizophrenia spectrum disorder $(n=93$, controls $)$ in the DPCRR. A posthoc analysis looked for a diagnosis of psychiatric or neurodevelopmental diseases (ICD-10 codes F20-F39, F70-F79, F80-F89, F90-F98) in the index child (serum sample collected during that pregnancy) from the DPCRR.

Cohort B: This included gestational week 14-18 mothers of children with mild or unspecified MR/ DPD (ICD-10: F70, F79, F80-F83.9, F88-F89.9) $(\mathrm{n}=171$, cases $)$ and mothers whose child had not 
received these diagnoses by the age at diagnosis of the case $(n=171$, controls) in the DPCRR.

Cohort C: This included gestational week 14-18 mothers of children with an autism spectrum disorder (ASD; ICD-10: F84.0, F84.1, F84.5, F84.8, F84.9) ( $\mathrm{n}=95$, cases) and mothers whose child had not received these diagnoses by the age at diagnosis of the case ( $n=95$, controls) in the DPCRR.

Cases and controls from all cohorts were matched on parity and age at delivery ( \pm 365 days) and also on gender of offspring in cohorts B and C.

\section{Antibody testing}

We screened samples for serum IgG-specific antibodies binding to NMDAR or CASPR2 (all cohorts) and Leucine Rich Glioma Inactivated 1(LGI1), alpha-amino-3-hydroxy-5-methyl-4-isoxazolepropionic acid receptor (AMPAR) and gamma-aminobutyric acid receptor b (GABABR) antibodies (cohort A only) using live immunofluorescent cell-based assays as performed in the Oxford laboratory for clinical diagnoses. ${ }^{910}$ These assays involve binding of serum IgG-specific antibodies to live human embryonic kidney cells transfected with cDNA encoding the different proteins. This cell-based assay uses a fluorescent-tagged secondary antibody (Alexa Fluor goat antihuman IgG, Invitrogen; A21090) that reacts with the human IgG heavy chains and the light chains of all classes of immunoglobulin. To confirm positivity and avoid detection of IgM antibodies (which are not transferred to the fetus), positive samples were additionally screened using a secondary antibody targeting the Fc region of IgG (goat antihuman Fc IgG; Thermo Scientific 31125) followed by detection with the appropriate fluorescent-tagged tertiary antibody (Alexa Fluor donkey antigoat IgG, Invitrogen A-11055). All samples were sent fully coded and decoding was done after reporting the results back to the Danish partners. Samples were only considered positive if agreed by two observers and also confirmed by the anti-IgG specific assay (two samples were insufficient for confirmation). This additional confirmatory step was done retrospectively for cohort A on coded samples. Seropositivity was scored visually on a non-linear scale from 0 to $4 .^{9} 10$

\section{Statistics}

In this exploratory study, Fisher's exact tests were used to compare differences as described in the text; $\mathrm{p}<0.05$ (two-tailed) was considered significant.

\section{Ethics}

The study was approved by the Danish Data Protection Agency and the Research Ethics Committee. All samples were coded, and experiments and analyses conducted blind to case status.

\section{RESULTS}

Cohort A: mothers with a diagnosis of psychosis after delivery and matched controls

Of all the pregnant women, 11 (5.8\%) had NMDAR antibodies (7/94 in mothers with subsequent psychosis vs 4/93 in control mothers $(\mathrm{p}=0.54))$ and $3(2 \%)$ had CASPR2 antibodies $(1 / 94$ and $2 / 93$, respectively), with no relationship of antibodies with later psychosis in the mothers $(\mathrm{p}=0.78$; see table 1$)$. All samples were negative for LGI1, AMPAR or GABABR antibodies.

However, when the records of the children were examined, a total of 28/187 (15\%; both psychosis mothers and controls) had a neuropsychiatric disorder diagnosis (see table 1 ). NMDAR or CASPR2 antibodies were present in 5/28 (18\%) of their mothers compared with only $9 / 159(6 \%)$ of the remaining mothers
Table 1 Results of cohort A according to neuropsychiatric diagnoses of the mother and offspring

\begin{tabular}{|c|c|c|c|}
\hline \multicolumn{4}{|c|}{$\begin{array}{l}\text { Mothers with subsequent psychosis and individually matched control } \\
\text { mothers }\end{array}$} \\
\hline & $\mathrm{N}$ & $\begin{array}{l}\text { CBA-positive } \\
\text { (NMDAR/CASPR2) }\end{array}$ & CBA-negative \\
\hline \multicolumn{4}{|l|}{ Mother } \\
\hline Psychosis & 94 & $8(7 / 1)$ & 86 \\
\hline No psychosis & 93 & $6(4 / 2)$ & 87 \\
\hline \multicolumn{4}{|l|}{ Offspring } \\
\hline Developmental disorder & 28 & $5(4 / 1)$ & 23 \\
\hline MR/DPD & 11 & $5(4 / 1)$ & 6 \\
\hline Schizophrenia & 3 & 0 & 3 \\
\hline Hyperkinetic disorders & 10 & 0 & 10 \\
\hline $\begin{array}{l}\text { Multiple diagnoses (not } \\
\text { including MR/DPD) }\end{array}$ & 4 & 0 & 4 \\
\hline $\begin{array}{l}\text { No neurodevelopmental } \\
\text { disorder }\end{array}$ & 159 & $9(7 / 2)$ & 150 \\
\hline
\end{tabular}

CASPR2, contactin-associated protein-like 2; MR/DPD, mental retardation and/ or disorders of psychological development; NMDAR, N-methyl-D-aspartate receptor. CBA = cell-based assay.

without such diagnoses in their offspring $(\mathrm{p}=0.04)$. Strikingly, $5 / 11(46 \%)$ mothers whose children received diagnoses in the category of MR/DPD had NMDAR antibodies $(n=4 ; 36 \%)$ or CASPR2 antibodies $(n=1,9 \%)$, compared with 9/176 (5\%; 7 NMDAR antibodies, 2 CASPR 2 antibodies) mothers of children without these diagnoses $(p=0.0004$; see table 2$)$. The association between maternal NMDAR antibody and neurodevelopmental disease was significant $(p=0.0018)$, but the results for CASPR2 antibodies (1/11 vs $2 / 176)$ did not reach significance $(p=0.17)$. None of the children had received a diagnosis in the autism spectrum.

\section{Cohort B: mothers of children with MR/DPD and matched controls}

Given the unpredicted finding of an association of maternal autoantibodies with MR/DPD, we then sought to corroborate this observation by studying a cohort of 171 mothers with children with MR/DPD and 171 control mothers. As expected ${ }^{11}$ for these diagnoses, there were more males than females (112 M, 59 F) in affected children and gender-matched controls.

NMDAR antibodies were not different between mothers with or without children with MR/DPD (10/171 (6\%) vs 9/171 $(5 \%))$. By contrast, there was a trend towards more frequent CASPR2 antibodies $(7 / 171 ; 4 \%)$ in the mothers of the children with MR/DPD compared with $1 / 171$ (1\%) of the control mothers $(p=0.067)$. The children of these seven mothers did not show any gender predominance (4 M, $3 \mathrm{~F}$ ). Two of the children had a diagnosis of mental retardation with or without impairment of behaviour, while the other five children had mixed specific developmental disorders, specific developmental disorders of motor function, speech articulation or reading disorders, or unspecified disorders of psychological development. Further information was restricted by Danish data protection regulations.

Figure 1A shows an example of a maternal serum binding to CASPR2-transfected cells, comparing with a control serum. Combining the data from cohorts A and B, CASPR 2 antibodies were identified in $8 / 182(4 \%)$ mothers with children with MR/ DPD and only $3 / 347(1 \%)$ in mothers of children without these diagnoses $(p=0.01$; figure $1 B)$. NMDAR antibodies were 
Table 2 Antibodies in maternal sera and their associations with MR/DPD in the offspring

\begin{tabular}{|c|c|c|c|}
\hline & \multicolumn{2}{|c|}{ Neuropsychiatric/developmental diagnosis of children } & \multirow[b]{3}{*}{ Fisher's $p$ value } \\
\hline & MR/DPD & No MR/DPD & \\
\hline & Positive/Total (\%) & Positive/Total (\%) & \\
\hline \multicolumn{4}{|c|}{ Cohort A: mothers with subsequent psychosis and individually matched control mothers } \\
\hline Either antibody & $5 / 11(45.5)$ & 9/176 (5.1) & 0.0004 \\
\hline CASPR2-Ab & $1 / 11(9.1)$ & 2/176 (1.1) & 0.17 \\
\hline NMDAR-Ab & $4 / 11(36.0)$ & $7 / 176(4.0)$ & 0.00518 \\
\hline \multicolumn{4}{|c|}{ Cohort B: mothers of children with mental retardation and other disorders of psychological development and individually matched control mothers } \\
\hline CASPR2-Ab & $7 / 171(4.1)$ & $1 / 171(0.6)$ & 0.067 \\
\hline NMDAR-Ab & 10/171 (5.8) & 9/171 (5.3) & $>0.99$ \\
\hline \multicolumn{4}{|l|}{ Combined analysis } \\
\hline CASPR2-Ab & $8 / 182(4.4)$ & $3 / 347(0.9)$ & 0.01 \\
\hline NMDAR-Ab & 14/182 (7.7) & 16/347 (4.6) & 0.17 \\
\hline
\end{tabular}

MR/DPD as defined here includes children with a mild or unspecified mental retardation with or without impairment of behaviour, specific developmental disorders of scholastic skills or other disorders of psychological development.

CASPR2, contactin-associated protein-like 2; MR/DPD, mental retardation and/or disorders of psychological development; NMDAR, N-methyl-D-aspartate receptor. Significant $p$ values are in bold.

present in 14/182 (8\%) mothers with children with MR/DPD and in $16 / 347(5 \%)$ other mothers $(\mathrm{p}=0.17$; table 2$)$.

\section{Cohort C: mothers of children with autism and matched controls}

During the later stages of this work, we became aware that others had identified CASPR2 antibodies in mothers of children with autism. ${ }^{12}$ However, in a cohort of samples from 95 mothers of children with a diagnosis of ASD, we did not find an excess of CASPR2 antibodies (one female, 1\%) compared with the mothers of children without this diagnosis (one male, one female, $2 \%, p>0.99$ ), and the overall positivity was very similar to that in the non-MR/DPD groups above (ie, around 1\%; table 3). NMDAR antibodies were also not different between mothers with or without children with ASD (2/95 (2\%) vs 3/95 (3\%), table 3).

\section{DISCUSSION}

The aetiology of intellectual disability (MR) or of specific developmental brain disorders is unknown in many cases. There are
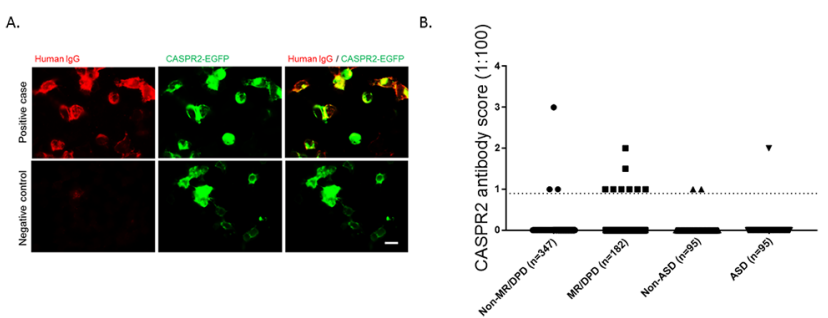

Figure 1 (A) Representative photomicrographs of the cell binding of a CASPR2-positive and a CASPR2-negative sample in the live cellbased assay (serum dilution 1:100; score 1.5). Scale bar: $20 \mu \mathrm{m}$. (B) CASPR2 antibody binding scores $(0-4$; negative $<1)$ in mothers of children with MR/DPD and control mothers (combined results of cohorts A and B) and in mothers of children with ASD and control mothers (cohort C). CASPR2 antibodies were five times more common in mothers of children given a diagnosis of MR/DPD, but not different between mothers of children with ASD and control mothers. Endpoint dilution antibody titres were generally low to modest, 1:100 (screening dilution) to 1:300, consistent with the scores shown. ASD, autism spectrum disorder; CASPR2, contactin-associated protein-like 2; MR/DPD, mental retardation and/or disorders of psychological development. genetic associations, reflecting multiple alleles of small effect, and rare cases with gene mutations or copy number variants. ${ }^{13}$ Maternal immunity is a relatively unexplored area. We linked a gestational serum biobank and patient register to show that maternal antibodies to a specific neuronal antigen, CASPR2, were associated with MR/DPD disorders in the progeny. Our results are the first to identify a specific target for potentially fetopathogenic antibodies in a large human gestational cohort.

Previous published data apply primarily to mothers of children diagnosed with $\mathrm{ASD},{ }^{3}$ rather than intellectual disability per se or the neurodevelopmental disorders identified here. Recently, CASPR2 antibodies were reported in $37 \%$ of a selected (brain-reactive) subgroup of mothers of autistic children compared with $8 \%-12 \%$ in the control groups; ${ }^{12}$ these authors demonstrated in an experimental model that a cloned maternal human CASPR2 antibody, applied during gestation, led to features of autism in male mouse offspring. However, we found no evidence for an increased prevalence of CASPR2 antibodies in the mothers of children with ASD, with only one CASPR2 antibody-positive patient in the autism cohort $(\mathrm{n}=95)$. Nevertheless, our results confirm that CASPR2 is a good candidate for antibody-associated neurodevelopmental disorders.

CASPR2 has an important role during neurodevelopment, being necessary for the correct formation of neural circuits through maturation of dendritic spines and control of dendritic arborisation. ${ }^{14}$ Variants of CNTNAP2, the gene encoding CASPR2, have been found in patients with ASD and in other neurodevelopmental disorders, such as psychosis, intellectual disability and speech impairment. ${ }^{15}$ Antibodies to CASPR2

Table 3 Antibodies in maternal sera and their associations with autism in the offspring

Cohort C: mothers of children with ASD and individually matched control mothers

\begin{tabular}{llll}
\hline & \multicolumn{2}{l}{ Psychiatric diagnosis of children } & \\
\cline { 2 - 3 } & ASD & No ASD & \\
\cline { 2 - 3 } & Positive/Total (\%) & Positive/Total (\%) & Fisher's p value \\
\hline CASPR2-Ab & $1 / 95(1.1)$ & $2 / 95(2.1)$ & $>0.99$ \\
NMDAR-Ab & $2 / 95(2.1)$ & $3 / 95(3.2)$ & $>0.99$ \\
\hline
\end{tabular}

ASD, autism spectrum disorder; CASPR2, contactin-associated protein-like 2; NMDAR, N-methyl-D-aspartate receptor. 
are present in adult patients with a variety of neurological syndromes. ${ }^{1}$ It seems likely that maternal CASPR2 antibodies transferred during pregnancy could impair the function of the protein during fetal brain development. Whether these antibodies are responsible for diagnoses of MR/DPD (or ASD) in the offspring, and the mechanisms involved, will require further careful analyses and animal models. Our data using a similar model system ${ }^{2}$ to that mentioned above ${ }^{12}$ also demonstrate longlasting changes in brain histology and behaviour in mice exposed to CASPR2 antibodies in utero (Ester Coutinho and Angela Vincent, under revision).

There are evident limitations and aspects for further study. Although NMDARs are important in intellectual development, and NMDAR antibodies are most frequent in young adult females, ${ }^{1}$ and occasionally cause postpartum psychosis, ${ }^{4}$ we did not find a reproducible association between gestational NMDAR antibodies and maternal psychosis or MR/DPD in the progeny, although NMDAR antibodies were more frequent overall in both groups. Regarding maternal CASPR2 antibodies, there was only a trend $(p=0.067)$ towards association with a diagnosis of MR/DPD in the offspring, unless we combined the data from both cohorts, which was not our a priori intention. Moreover, the levels of antibodies were often low (see figure 1 legend), unlike the higher titres (>1:500) typically found in patients with autoimmune encephalitis; ${ }^{16}$ this can be partly explained by decreased immunity in pregnancy, increased plasma volume and transfer of IgG to the fetus during pregnancy. ${ }^{17}$ Unfortunately, at these low levels, confirmatory tests for CASPR2 antibodies, such as immunohistochemistry or binding to live neurons, are not positive in our hands (Ester Coutinho, Angela Vincent unpublished results). Third, the CASPR2 antibodies were also found in four control mothers overall, indicating that there are likely other factors that determine their pathogenicity, such as expression levels of the neonatal Fc receptor, timing of the antibodies in relation to the maximum potential damage to the developing brain or the existence of additional proinflammatory cytokines. Fourth, phenotypic overlap between ASD and $\mathrm{MR} / \mathrm{DPD}$ can occur, but this was not possible to explore due to data protection.

Despite the limitations, these data should stimulate further research, both epidemiological and experimental, into the role of CASPR2 and other potentially fetopathogenic maternal antibodies in disorders of brain development. This study found an association between maternal CASPR2 antibodies and a diagnosis of MR/DPD in the progeny, but it is possible that other antibodies targeting other, yet unknown, neuronal antigens might be present in the maternal sera. The association found in this study, although interesting and potentially relevant, needs further validation in other patient cohorts, and the pathogenic potential of CASPR2 antibodies needs to be established in appropriate animal models, as in the study of Brimberg et $\mathrm{al}^{12}$ and our own (Ester Coutinho and Angela Vincent, under revision). Only then may we be able to translate these findings into clinical practice, and possibly offer prenatal screening to mothers with multiple affected pregnancies, in line with other antibody-mediated neonatal and neurodevelopmental disorders.

\section{Author affiliations}

'Nuffield Department of Clinical Neurosciences, University of Oxford, Oxford, UK ${ }^{2}$ Department of Economics and Business Economics, National Centre for Registerbased Research, Aarhus University, Aarhus, Denmark

${ }^{3}$ PSS YCH, The Lundbeck Foundation Initiative for Integrative Psychiatric Research, Aarhus, Denmark

${ }^{4}$ Mental Health Centre Copenhagen, University of Copenhagen, Faculty of Health Sciences, Copenhagen, Denmark
${ }^{5}$ Department of Congenital Disorders, Danish Centre for Neonatal Screening, Statens Serum Institut, Copenhagen, Denmark

${ }^{6}$ Centre for Integrated Register-Based Research (CIRRAU), Aarhus University, Aarhus, Denmark

${ }^{7}$ Department of Psychiatry, University of Oxford, Oxford, UK

${ }^{8}$ Oxford Health NHS Foundation Trust, Oxford, Oxfordshire, UK

Contributors EC and AV conceptualised the study. EC and AV were responsible for methodology. EC, LJ, MGP and MEB conducted the investigation. AV obtained resources. PH, PBM, BN-P and AV supervised the study. EC and AV wrote the manuscript. All authors revised the manuscript.

Funding EC is funded by Programme for Advanced Medical Education, Fundação Calouste Gulbenkian. This work was also supported by the Stanley Medical Research Institute.

Competing interests $A V$ and the University of Oxford hold a patent for LG11 and CASPR2 antibodies, licensed to Euroimmun AG, and receive a proportion of royalties for autoimmune encephalitis diagnostics.

Patient consent Historical cohort under Danish research ethics.

Ethics approval Danish Data Protection Agency and the Research Ethics Committee.

Provenance and peer review Not commissioned; externally peer reviewed.

Open Access This is an Open Access article distributed in accordance with the Creative Commons Attribution Non Commercial (CC BY-NC 4.0) license, which permits others to distribute, remix, adapt, build upon this work non-commercially, and license their derivative works on different terms, provided the original work is properly cited and the use is non-commercial. See: http://creativecommons.org/ licenses/by-nc/4.0/

(c) Article author(s) (or their employer(s) unless otherwise stated in the text of the article) 2017. All rights reserved. No commercial use is permitted unless otherwise expressly granted.

\section{REFERENCES}

1 Crisp SJ, Kullmann DM, Vincent A. Autoimmune synaptopathies. Neuroscience Nature Reviews 2016;17:103-17

2 Dalton P, Deacon R, Blamire A, et al. Maternal neuronal antibodies associated with autism and a language disorder. Ann Neurol 2003;53:533-7.

3 Braunschweig D, Krakowiak P, Duncanson P, et al. Autism-specific materna autoantibodies recognize critical proteins in developing brain. Trans/ Psychiatry 2013;3:e277.

4 Bergink V, Armangue T, Titulaer MJ, et al. Autoimmune encephalitis in postpartum psychosis. Am J Psychiatry 2015;172:901-8.

5 Hameed MA, Lewis AJ. Offspring of parents with schizophrenia: a systematic review of developmental features across childhood. Harv Rev Psychiatry 2016;24:104-17.

6 Pedersen CB. The danish civil registration system. Scand J Public Health 2011;39(7 Suppl):22-5.

7 Pedersen MG, Stevens H, Pedersen CB, et al. Toxoplasma infection and later development of schizophrenia in mothers. Am J Psychiatry 2011;168:814-21.

8 Mors O, Perto GP, Mortensen PB. The danish psychiatric central research register. Scand J Public Health 2011;39(7 Suppl):54-7.

9 Irani SR, Bera K, Waters P, et al. N-methyl-D-aspartate antibody encephalitis: temporal progression of clinical and paraclinical observations in a predominantly nonparaneoplastic disorder of both sexes. Brain 2010;133(Pt 6):1655-67.

10 Irani SR, Alexander S, Waters P, et al. Antibodies to Kv1 potassium channel-complex proteins leucine-rich, glioma inactivated 1 protein and contactin-associated protein-2 in limbic encephalitis, Morvan's syndrome and acquired neuromyotonia. Brain 2010;133:2734-48.

11 Huang J, Zhu T, Qu Y, et al. Prenatal, perinatal and neonatal risk factors for intellectual disability: a systemic review and Meta-Analysis. PLoS One 2016;11:e0153655.

12 Brimberg L, Mader S, Jeganathan V, et al. Caspr2-reactive antibody cloned from a mother of an ASD child mediates an ASD-like phenotype in mice. Mol Psychiatry 2016;21:1663-71.

13 Vissers LE, Gilissen C, Veltman JA. Genetic studies in intellectual disability and related disorders. Nat Rev Genet 2016;17:9-18.

14 Anderson GR, Galfin T, Xu W, et al. Candidate autism gene screen identifies critical role for cell-adhesion molecule CASPR2 in dendritic arborization and spine development. Proc Natl Acad Sci U S A 2012;109:18120-5.

15 Rodenas-Cuadrado P, Ho J, Vernes SC. Shining a light on CNTNAP2: complex functions to complex disorders. Eur J Hum Genet 2014:22:171-8.

16 Irani SR, Pettingill P, Kleopa KA, et al. Morvan syndrome: clinical and serological observations in 29 cases. Ann Neurol 2012;72:241-55.

17 Malek A, Sager R, Kuhn P, et al. Evolution of maternofetal transport of immunoglobulins during human pregnancy. Am J Reprod Immunol 1996:36:248-55. 